\title{
Positivity of the virial coefficients in lattice dimer models and upper bounds on the number of matchings on graphs
}

\author{
P. Butera, ${ }^{1, *}$ P. Federbush, ${ }^{2, \dagger}$ and M. Pernici ${ }^{3, \dagger}$ \\ ${ }^{1}$ Dipartimento di Fisica Universita' di Milano-Bicocca \\ and \\ Istituto Nazionale di Fisica Nucleare \\ Sezione di Milano-Bicocca \\ 3 Piazza della Scienza, 20126 Milano, Italy \\ ${ }^{2}$ Department of Mathematics \\ University of Michigan \\ Ann Arbor, MI 48109-1043, USA \\ ${ }^{3}$ Istituto Nazionale di Fisica Nucleare \\ Sezione di Milano \\ 16 Via Celoria, 20133 Milano, Italy
}

(Dated: September 19, 2018)

\begin{abstract}
Using a simple relation between the virial expansion coefficients of the pressure and the entropy expansion coefficients in the case of the monomer-dimer model on infinite regular lattices, we have shown that, on hypercubic lattices of any dimension, the virial coefficients are positive through the 20th order. We have observed that all virial coefficients so far known for this system are positive also on infinite regular lattices with different structure. We are thus led to conjecture that the virial expansion coefficients $m_{k}$ are always positive.

These considerations can be extended to the study of related bounds on finite graphs generalizing the infinite regular lattices, namely the finite grids and the regular biconnected graphs. The validity of the bounds $\Delta^{k} \ln (i ! N(i)) \leq 0$ for $k \geq 2$, where $N(i)$ is the number of configurations of $i$ dimers on the graph and $\Delta$ is the forward difference operator, is shown to correspond to the positivity of the virial coefficients.

Our tests on many finite lattice graphs indicate that on large lattices these bounds are satisfied, giving support to the conjecture on the positivity of the virial coefficients. Moreover, in an exhaustive survey of some classes of regular biconnected graphs with a not too large number $v$ of vertices, we observe only few violations of these bounds. We conjecture that the frequency of the violations vanishes as $v \rightarrow \infty$.

Using an inequality by Heilmann and Lieb, we find rigorous upper bounds on $N(i)$ valid for arbitrary graphs and for regular graphs. The similarity between this inequality and the one conjectured above suggests that one study the stricter inequality $m_{k} \geq \frac{1}{2 k}$ for the virial coefficients, which is valid for all the known coefficients of the infinite regular lattice models.
\end{abstract}

PACS numbers: $05.50 .+\mathrm{q}, 64.60 . \mathrm{De}, 75.10 . \mathrm{Hk}, 64.70 . \mathrm{F}-, 64.10 .+\mathrm{h}$

Keywords: Dimer problem, graph entropy, upper bounds on matchings in regular graphs

\section{INTRODUCTION}

The virial series-expansion coefficients of the pressure have been computed through relatively high orders for the monomer-dimer (MD) models on various infinite regular lattices. Presently, they are tabulated[1] through order 19 for the tetrahedral lattice and through order 7 for the hexagonal lattice 2. Moreover, they are known for the triangular and the face-centered-cubic lattices through the orders 14 and 10 respectively [3] . In the case of the linear lattice [5]6] and of the Bethe lattice 78 , they are all known. We have recently computed 9 the expansions through the order 24 for the $b c c$ lattices in $d=3,4,5,6,7$, and for the (hyper)-simple-cubic lattices, through order 24 in the case of the square, cubic and $4 d$ lattices, through the orders 22 and 21 in dimensions $d=5$ and 6 respectively, and finally[10] in general dimensions $d>6$ through the order 20 .

Long ago, Heilmann and Lieb 11/12 have studied the MD models also on finite graphs. They have shown that the zeroes of the matching generating polynomial $M(z)=\sum N(i) z^{i}$ of a graph lie only on the real negative axis of the complex $z$ plane. For the MD gas on a finite lattice, this implies the absence of any phase-transition in the thermodynamic limit.

In this paper, we show that the first 20 coefficients of the virial expansion on hypercubic infinite regular lattices of any dimension are positive. With the knowledge that all the coefficients computed so far for the virial expansions of the MD models on all infinite regular lattices are positive, we are led to conjecture that all the virial coefficients are positive for the infinite regular lattice models.

Using the definition of the graph dimer entropy 14, we argue that for a finite regular graph the bounds which 
correspond to the positivity of the virial coefficients $m_{k}$ for infinite regular lattices are

$$
\Delta^{k} \ln (i ! N(i)) \leq 0
$$

with $k=2, \ldots, \nu$ and $i=0, \ldots, \nu-k$, where $\nu$ is the matching number of $G$, i.e. the maximum number of pairwise disjoint edges of $G$.

We have systematically tested the validity of Eq. (1) within two classes of finite graphs related to infinite regular lattices. The first class consists of the graphs induced by finite grids, the second consists of the biconnected regular simple graphs. The tests of Eq. (1) for the latter class have been performed by exhaustively generating the biconnected regular graphs having a not too large number of vertices $v$, with the aid of the Nauty program[15] via the Sage interface 16 .

The results of our survey are summarized in a set of schematic tables reported in the fourth Section. We have firstly restricted our attention to the class of finite lattices, being interested in finding sequences of finite lattices with no violations of the bounds in Eq. (1). This would indicate that all the virial coefficients are positive for the corresponding infinite regular lattices. Even though the bounds in Eq. (1) have been derived only for regular graphs, it is however interesting to consider them also in the case of finite grids with open boundary conditions $(b c)$, (which clearly are not regular on the boundary).

In summary, we found violations only for "narrow" lattices. This fact supports the conclusion that the bounds are not violated in the limit of infinite lattices.

We then turned to the more general class of the bipartite biconnected regular simple graphs. For the 3-regular graphs, we were able to test these bounds for $v \leq 30$ vertices. We have observed a few violations of the bounds for $v \geq 18$, but the frequency of these violations decreases regularly for $v \geq 18$.

In the case of the bipartite biconnected 4-regular graphs, we have tested Eq. (1) for $v \leq 22$. We have observed a single violation for $v=20$. The frequency of the violations is even lower for $v=22$.

In the case of the bipartite biconnected $r$-regular graphs with $r>4$, we could carry our tests only for $v \leq 20$, finding a single violation, that occurs in a 5-regular graph.

When considering the non-bipartite biconnected 3-regular graphs, we observed few violations for $v \leq 22$, starting from $v=12$; again the frequency of the violations decreases regularly after the first violation, for even values of $v \geq 12$. Notice that there are no such graphs with $v$ odd.

In the case of the non-bipartite biconnected 4-regular graphs, the frequency of the violations decreases regularly for even $v$ after the first violation and a similar trend is observed for odd $v$. We could complete the tests only for $v \leq 17$. In this case one has to consider over 80 million graphs.

Based on the results of our survey, we are led to conjecture that, for biconnected regular graphs, the bounds are violated with a frequency that vanishes as $v \rightarrow \infty$.

Let us now observe that the validity of the bounds of Eq. (1) in the case $k=2$ follows from the Heilmann-Lieb inequality Eq. (4) in [11. Using this Heilmann-Lieb inequality, we shall derive rigorous upper bounds for $N(i)$ of general graphs. In the case of regular graphs they are stricter than those in [17] in a region with small dimer density; in the case of general graphs, we obtain an upper bound for the matching generating polynomial which is stricter than the one found in [18].

The (rigorous) Heilmann-Lieb inequality has the form

$$
\Delta^{k} \ln (i !(n-i) ! N(i)) \leq 0
$$

with $n=\left[\frac{v}{2}\right]$ and $k=2$. The similarity between this inequality and Eq. (1) led us to investigate Eq. (2) also for $k \geq 2$ and to conjecture for infinite regular lattices the stricter bound $m_{k} \geq \frac{1}{2 k}$, which indeed is satisfied by all known virial coefficients. As in the previous case, we have investigated how well the bounds Eq. (2), with $k \geq 2$, are satisfied by finite grids and regular biconnected graphs.

The tests of Eq. (2), with $k \geq 2$, on lattice graphs give more violations than for Eq. (1).

Therefore the examination of these lattices still gives some indication, though not as clear as in the case of Eq. (1), that the bounds Eq. 22, with $k \geq 2$, are satisfied by the corresponding infinite lattices and that the virial coefficients are positive.

For the general graphs produced with the aid of Nauty, we find similar results as with the bounds of Eq. (1), although we observe more violations.

We have also shown that, for any $v$, the bounds of Eqs. (1, 2) are satisfied by the approximate distribution of bipartite regular random-graph obtained in [19. In the case of bipartite regular biconnected graphs, we argue that a conjecture on the entropy for these graphs, made in [19/25], implies the conjecture that for $v \rightarrow \infty$ the frequency of the violations tends to zero.

In all the tests performed on regular biconnected graphs (over more than 300 million graphs), Eq. (2) is valid for $k=3$. We shall discuss also the corresponding upper bounds on $N(i)$; the examples considered indicate that in the 
case of bipartite regular biconnected graphs they are less strict than the Upper Matching conjecture (UMC) [19], when the latter applies.

Let us mention that while the UMC conjecture has not been yet proven, the lower asymptotic matching conjecture stated in [19] has been proven in [20] and [21].

The paper is organized as follows. In the second Section, using a simple relation between the coefficients of the virial expansion of the pressure and the expansion coefficients of the dimer entropy, we prove that on hypercubic lattices the virial coefficients through order 20 are positive for generic $d$. In the third Section, we derive Eq. (1), obtain rigorous upper bounds for $N(i)$ and discuss Eq. (2). The fourth Section summarizes the results of the graph tests for a variety of lattices and graphs. In Appendix $\mathrm{A}$ we give the virial coefficients for $d=2,3$ through order 24. In Appendix B, the validity of Eq. (1), and of Eq. (2) is proven for a few classes of graphs and for two average distributions. In Appendix $\mathrm{C}$ a formula for the bound on $N(i)$ is deduced from the inequalities of Eq. (2).

\section{POSITIVITY OF VIRIAL COEFFICIENTS}

The combinatorial-statistical properties of a MD system on a $r$-regular lattice are usually described in the grandcanonical formalism of statistical mechanics, in which the pressure is defined as

$$
\lim _{v \rightarrow \infty} \frac{1}{v} \ln \Xi_{v}(z)=P(z)=\sum_{i} b_{i} z^{i} .
$$

Here $\Xi_{v}(z)$ is the grand-canonical partition function for a $v$-site lattice and $z$ is the activity. The dimer density is then

$$
\rho(z)=z \frac{d P}{d z}=\sum_{i=1}^{\infty} i b_{i} z^{i}
$$

Setting $p=2 \rho$, and solving Eq. (4) for $z=z(p)$, we can express the pressure in terms of $p$

$$
P(p)=p / 2+\sum_{k=2}^{\infty} m_{k} p^{k}
$$

This is the virial expansion. The entropy is defined by

$$
\lambda(p)=-\rho(z) \ln z+P(z)
$$

from Eqs.(4) and (6) one gets 1013

$$
\frac{d \lambda}{d p}=-\frac{\ln z}{2}
$$

Using the expansion 10 26

$$
\lambda(p)=R(p)+\sum_{k=2}^{\infty} a_{k} p^{k}
$$

where

$$
R(p)=\frac{1}{2}(p \ln (r)-p \ln p-2(1-p) \ln (1-p)-p)
$$

and $r$ is the lattice coordination number, from Eq. (7) one has

$$
\ln z=\ln \left(\frac{p}{r(1-p)^{2}}\right)-2 \sum_{k=2}^{\infty} k a_{k} p^{k-1}
$$

Substituting in Eq. (6) $\ln z$ from Eq. (10) and $\lambda$ from Eqs. (8) 9) one obtains

$$
P=-\ln (1-p)-\frac{p}{2}+\sum_{k=2}^{\infty}(1-k) a_{k} p^{k}
$$


so that a simple relation is obtained between the coefficients $m_{k}$ of the virial expansion and the coefficients $a_{k}$ of the entropy expansion

$$
m_{k}=(k-1)\left(\frac{1}{k(k-1)}-a_{k}\right)
$$

In the case of hypercubic lattices in any dimension $d$ the coefficients $a_{k}$ have been computed in [10] through the order 20; in that reference in the cases $d=2,3,4$ there are the values through order 24 . In appendix A we list the corresponding virial coefficients for $d=2,3$.

On these lattices, we can express the coefficients of the pressure, of the virial and the entropy expansions as simple polynomials in the variable $1 / d$. Using the expressions for $a_{k}$ with $k \leq 20$ in Ref. [10] to examine the real roots of $m_{k}$, reported in Table $\mathbb{I}$, and observing that, for large $d$, the leading coefficient of $m_{k}$ is positive, it follows that $m_{k}$ is positive for any integer $d$ with $d \geq 1$.

For example for $k=10$ one has

$$
m_{10}=\frac{1024 d^{9}-35712 d^{4}+123240 d^{3}-118260 d^{2}-36990 d+67721}{10240 d^{9}}
$$

with the three real root given in Table $\mathbb{I}$, dividing the real axis in regions in which $m_{10}$ has signs,,,-+-+ ; the positive integer values of $d$ occur in the positive regions.

TABLE I: Real roots of $m_{k}$ for $k \leq 20$

\begin{tabular}{|c|c|c|c|c|}
\hline$k=2$ & 0.25 & & & \\
$k=3$ & -0.354 & 0.354 & & \\
$k=4$ & -0.859 & & & \\
$k=5$ & none & & & \\
$k=6$ & -0.239 & & & \\
$k=7$ & -2.032 & 0.848 & & \\
$k=8$ & -1.796 & -0.557 & 0.859 & \\
$k=9$ & 1.044 & 1.257 & & \\
$k=10$ & -0.655 & 1.029 & 1.313 & \\
$k=11$ & -3.404 & 0.998 & & \\
$k=12$ & -3.241 & -0.125 & 0.997 & \\
$k=13$ & 1.000097 & 1.725 & & \\
$k=14$ & 0.9994 & & & \\
$k=15$ & -4.657 & 0.999997 & & \\
$k=16$ & -4.617 & 1.000004 & 1.801 & \\
$k=17$ & 1.000000085 & 1.963 & & \\
$k=18$ & 0.99999993 & & & \\
$k=19$ & -5.852 & 0.999999998 & 2.005 & 2.396 \\
$k=20$ & -5.879 & 1.000000001 & 1.993 & \\
\hline
\end{tabular}

As remarked in the introduction, all the virial expansion coefficients so far computed for the lattice dimer models are positive. This leads us to conjecture that they are all positive on all infinite regular lattices.

Let us remark that on any lattice with coordination number $r$ one has

$$
a_{k}=\frac{r^{1-k}}{2 k(k-1)}
$$

for $k$ less than the girth (i.e. the number of edges in a shortest cycle) of the lattice graph; in particular this is true to all orders in the case of the Bethe lattice [78].

Note that from the assumption that the virial coefficients are all positive, one gets an upper bound on the $a_{k}$,

$$
a_{k} \leq \frac{1}{k(k-1)}
$$




\section{AN ARGUMENT FOR THE BOUNDS CONJECTURED IN EQ. (1) AND EQ. (2).}

In Ref.14, we introduced the Newton series for the dimer entropy of a graph, in terms of the quantities

$$
d(i)=\ln \left(\frac{N(i)}{N(1)^{i}}\right)-\ln \left(\frac{\bar{N}(i)}{\bar{N}(1)^{i}}\right)
$$

Here $N(i)$ is the number of configurations of $i$ dimers on the graph $G$ with $v$ vertices and $\bar{N}(i)$ given by

$$
\bar{N}(i)=\frac{\bar{v} !}{(\bar{v}-2 i) ! i ! 2^{i}}
$$

is the number of configurations of $i$ dimers on the complete graph on $\bar{v} \equiv 2 \nu$ vertices, where $\nu$ is the matching number of $G$. If the graph $G$ has a perfect matching $v=\bar{v}$.

For a graph that satisfies the "graph positivity" property introduced in Ref.[14] one has

$$
\Delta^{k}[d](i) \geq 0
$$

where $k=0, \ldots, \nu$ and $i=0, \ldots, \nu-k$. The validity of these bounds for all allowed $k$ and $i$ is in fact equivalent to their validity for all allowed $k$ and $i=0$.

If the "graph positivity" conjecture of Ref. [14] is true, then eq.17) holds for almost all regular biconnected bipartite graphs (in a reasonable sense). More precisely, if this conjecture is true, then for each $r$ the fraction of $r$-regular biconnected bipartite graphs with $v$ vertices that satisfy eq. (17) tends to 1 as $v \rightarrow \infty$. This positivity property is often satisfied also in non-regular bipartite graphs, while it is usually not satisfied by non-bipartite graphs.

We have a similar situation here. Based upon the conjecture that all virial coefficients are positive for dimer models on infinite regular lattices, we state the following conjecture: the fraction of regular biconnected graphs with $v$ vertices that satisfy

$$
\Delta^{k}[d](i) \leq \Delta^{k} \ln \frac{(\bar{v}-2 i) ! \bar{v}^{2 i}}{\bar{v} !}
$$

tends to 1 as $v \rightarrow \infty$, when $k=2, \ldots, \nu$ and $i=0, \ldots, \nu-k$. The validity of these bounds for all allowed $k$ and $i$ is in fact equivalent to their validity for all allowed $k$ and $i=0$. Unfortunately, we do not know how to state mathematically how preponderantly this property holds for finite $v$. This will be seen "experimentally" in the following sections. We now trace the path which leads from positivity of the virial series coefficients to Eq. (18) in the case of finite lattices.

With $p \approx \frac{2 i}{v}$, in the limit of large $v$ one has (see Eq. (11) in [14])

$$
\frac{1}{v} d(i) \rightarrow \sum_{k=2}^{\infty} a_{k} p^{k}
$$

We eliminate $a_{k}$ using Eq. 12 and observe that

$$
\frac{1}{v} \ln \frac{(v-2 i) ! v^{2 i}}{v !} \rightarrow \sum_{k=2}^{\infty} \frac{p^{k}}{k(k-1)}
$$

Thus we get

$$
\frac{1}{v} d(i)-\frac{1}{v} \ln \frac{(v-2 i) ! v^{2 i}}{v !} \rightarrow-\sum_{k=2}^{\infty} \frac{1}{(k-1)} m_{k} p^{k}
$$

Using $\frac{v}{2} \Delta \approx \frac{d}{d p}$ [14, assuming that the $m_{k}$ are positive and that $\frac{\bar{v}}{v} \rightarrow 1$ for $v \rightarrow \infty$, we are led to Eq. [18), in which we used $\bar{v}$ instead of $v$, motivated by the fact that the tests of Eq. 18 have fewer violations this way. The assumption that $\frac{\bar{v}}{v} \rightarrow 1$ is verified for biconnected 3-regular graphs and for regular bipartite graphs, since in these cases there exists a perfect matching (see [27] and references within). In fact all the biconnected regular graphs systematically examined in next section have $\nu=n$, where $n=\left[\frac{v}{2}\right]$. 
Using Eqs.15, 16) we can rewrite Eq. (18) as

$$
\Delta^{k} \ln N(i) \leq \Delta^{k}\left(i \ln \left(\frac{N(1) \bar{v}}{\bar{v}-1}\right)-\ln (i !)\right)
$$

For $k \geq 2$, these bounds reduce to Eq. (1).

From the bound Eq. (4) in [11], setting in that equation $Z_{i}=N(n-i)$ and $M=n$, with $n \equiv\left[\frac{v}{2}\right]$, we get

$$
\Delta^{2} \ln N(i) \leq \ln \frac{(i+1)(n-i-1)}{(i+2)(n-i)}=-\Delta^{2} \ln (i !(n-i) !) .
$$

It follows that, for $k=2$, the bounds of Eq. (1) are valid for any graph.

Initially Eq. (18) would seem to hold only for the graphs, for example the periodic cubical graphs, whose limits are used to get the given lattice functions. But we will try to apply Eq. (18), or equivalently Eq. (1), to regular biconnected graphs and to finite lattices.

\section{A. Rigorous upper bounds on the number of matchings}

The bound Eq. 23) follows from the fact that all roots of the matching generating polynomial $M(z)=\sum_{i=0}^{\nu} N(i) z^{i}$ are negative[12], so that the quantities $P_{\nu}(i)=N(i) /\left(\begin{array}{c}\nu \\ i\end{array}\right)$ satisfy the Newton's inequalities $P(i+1) P(i-1) \leq P(i)^{2}$, or equivalently

$$
\Delta^{2} \ln P(i) \leq 0
$$

for $i=0, . ., \nu-2$. Using these inequalities and $\nu \leq n$, where $n=\left[\frac{v}{2}\right]$, it is easy to see that also

$$
P(i)=\frac{N(i)}{\left(\begin{array}{c}
n \\
i
\end{array}\right)}
$$

satisfies them for $i=0, . ., n-2$.

Eq. (24) leads to the bounds (see [28] and Eq. 253] of Appendix C for the quantity $g(i)=\ln P(i)$ with $k=2$ )

$$
P(i) \leq\left(\frac{P\left(i_{0}+1\right)}{P\left(i_{0}\right)}\right)^{i-i_{0}} P\left(i_{0}\right)
$$

for $i \geq i_{0}$. In the case of general graphs, we can apply Eq. 26) with $i_{0}=0$, using $N(0)=1$ and $N(1)=E$, where $E$ is the number of edges of the graph.

In the following we will obtain a series of bounds based on Eq. 53 with given $k$ and $i_{0}$; we will denote such bounds as "BXk.i. $i_{0}$ ", where B stands for Bound, X in G for a General graph, R for a Regular graph and B for Bipartite regular graph; $k$ and $i_{0}$ are indices in Eq. (53). The case $k=2$ corresponds to the Heilmann-Lieb inequality, holding for all graphs. The case $k=3$, discussed in the next subsection, is conjectural; to emphasize that we add the letter c to the name of the bound in the case $k=3$.

The choice of $i_{0}$ depends on the known $N(i)$ for a category of graphs; for a general graph one has $N(0)=1$ and $N(1)=E$.

Thus we get an upper Bound for General graphs that we call BG2.0 to indicate that it follows from Eq. 22 with $k=2, i_{0}=0$,

$$
N(i) \leq(E / n)^{i}\left(\begin{array}{c}
n \\
i
\end{array}\right)
$$

From this a simple bound for the matching generating polynomial follows

$$
|M(z)|=\left|\sum_{i=0}^{n} N(i) z^{i}\right| \leq\left(1+\frac{E|z|}{n}\right)^{n}
$$

Using $P_{\nu}(i)$ instead of $P(i)$ one gets Eq. (28) with $n$ replaced by $\nu$; this stricter bound has been derived in [18. 
For $E$ fixed, $v \leq 2 E$. For the Hosoya index $Z=M(1)$, introduced 29 in theoretical chemistry to characterize the topological structure of large molecules, when $v=2 E$ the bound Eq. $(28)$ is saturated by the graph $n K_{2}$. As $v \rightarrow \infty$, for $z=1$ the bound Eq. (28) tends to the bound $Z<e^{E}$, first derived in [30].

In the following we will derive other bounds for $P(i)$; one could write down analogous bounds using $P_{\nu}(i)$, when $\nu<n$.

For a graph with $E$ edges and vertices of degrees $\delta_{i}$, we have

$$
N(2)=\left(\begin{array}{c}
E \\
2
\end{array}\right)-\sum_{i=1}^{v}\left(\begin{array}{c}
\delta_{i} \\
2
\end{array}\right)
$$

with

$$
\sum_{i=1}^{v} \delta_{i}=2 E
$$

Define $r=\left[\frac{2 E}{v}\right]$ and $h=2 E-r v$, then one has $0 \leq h<v$. Defining $\delta_{i}=r+k_{i}$, we get $\sum_{i=1}^{v} k_{i}=h$ and

$$
\sum_{i=1}^{v} \delta_{i}^{2}=v r^{2}+2 r \sum_{i=1}^{v} k_{i}+\sum_{i=1}^{v} k_{i}^{2} \geq v r^{2}+(2 r+1) \sum_{i=1}^{v} k_{i}
$$

so that

$$
\sum_{i=1}^{v} \delta_{i}^{2} \geq v r^{2}+(2 r+1)(2 E-r v)
$$

It follows that using the bound Eq. (26) with $i_{0}=1$, we arrive at the bound BG2.1, slightly tighter than BG2.0 for $i>0$

$$
N(i) \leq \frac{E}{n}\left(\frac{2 N_{m}(2)}{(n-1) E}\right)^{i-1}\left(\begin{array}{c}
n \\
i
\end{array}\right)
$$

where $N_{m}(2)$ is the maximum value of $N(2)$

$$
N_{m}(2)=\frac{E(E+1)-v r^{2}-(2 r+1)(2 E-r v)}{2}
$$

with

$$
r \equiv\left[\frac{2 E}{v}\right]
$$

In the case of the BG2.1 bound, for the matching generating polynomial we obtain

$$
|M(z)| \leq 1+\frac{(n-1) E^{2}}{2 n N_{m}(2)}\left(\left(1+\frac{2|z| N_{m}(2)}{(n-1) E}\right)^{n}-1\right)
$$

As an example, consider the logarithm of the Hosoya index $Z$ for a general graph with $v=60$ and $E=110$. Then the upper bound BG2.0 for this quantity has the value 46.2 while the bound BG2.1 has the value 45.6.

In [17] it has been shown that for a regular graph with even $v$, the $N(i)$ satisfy the following bounds for $0 \leq i \leq n$; the bound

$$
N(i) \leq 2^{-i}\left(\begin{array}{c}
2 n \\
i
\end{array}\right) r^{i}
$$

which is tight in the region of low dimer density and

$$
N(i) \leq\left(\begin{array}{c}
2 n \\
2 i
\end{array}\right)(r !)^{\frac{i}{r}}
$$


which is tight in the region of high dimer density. In 31] another bound tight in the region of high dimer density is given for $i<n$

$$
N(i) \leq \exp \left(\frac{v}{2}\left(p \ln (r)-p \ln (p)-2(1-p) \ln (1-p)-p+\frac{\ln (r)}{r-1}\right)\right)
$$

where $p=\frac{i}{n}$. We shall denote by BR, the bound for the regular graphs that is the minimum among these three bounds. For $r$ constant and $n$ large, the bound Eq. (27) improves Eq. (37) by a factor slightly larger than

$$
\frac{(1-p)^{n-i+\frac{1}{2}}}{\left(1-\frac{p}{2}\right)^{2 n-i+\frac{1}{2}}}
$$

for $p$ not close to 0 or 1 ; for instance if $i=\frac{n}{2}$, this factor is $1.09^{n}$. Therefore a stricter bound (that we denote as BR2.0), is obtained combining Eq. 27) and Eq. (38). A slightly stricter bound (that we call BR2.1) is obtained combining Eq. (33) and Eq. (38).

In the case of regular bipartite graphs the following inequalities are known [17] for $0 \leq i \leq n$

$$
N(i) \leq\left(\begin{array}{c}
n \\
i
\end{array}\right) r^{i}
$$

giving a tight bound in the region of low dimer density and

$$
N(i) \leq\left(\begin{array}{c}
n \\
i
\end{array}\right)^{2}(r !)^{\frac{i}{r}}
$$

giving a tight bound in the region of high dimer density. They lead to the bound Eq. (3.3) in [17] (that we denote as BB). The bound Eq. (27) is the same as Eq. (40) for these graphs. We can obtain a bound (denoted as BB2.2), which is stricter than $\mathrm{BB}$, by using Eq. (26) with $i_{0}=2$, and observing that

$$
N(2)=\frac{n r(n r-2 r+1)}{2}
$$

and 32

$$
N(3)=\left(\begin{array}{c}
n r \\
3
\end{array}\right)-2 n\left(\begin{array}{l}
r \\
3
\end{array}\right)-n r(r-1)^{2}-2 n\left(\begin{array}{l}
r \\
2
\end{array}\right)(n r-3 r+2)
$$

Using Eq. (26) with $i_{0}=3$, using $N(3)$ and the maximum value of $N(4)$, given by 32 .

$$
N_{m}(4)=\frac{n^{4} r^{4}}{24}+\frac{n^{3} r^{3}}{4}(1-2 r)+\frac{n^{2} r^{2}}{24}\left(19-60 r+52 r^{2}\right)+n r\left(\frac{5}{4}-5 r+7 r^{2}-\frac{7 r^{2}}{2}\right)+\frac{r n(r-1)^{2}}{4}
$$

we get a bound slightly better than choosing $i_{0}=2$. For $r$ fixed, $v$ large, and $i_{0}=3$, the bound Eq. 26 for $N(i)$ is smaller than the bound Eq. 40 by roughly a factor $\exp \left(\left(3-\frac{3}{r}\right) \frac{i-2}{n}\right)$. For instance with $i=\frac{n}{2}$ and $n$ large, the bound is smaller by a constant factor $\exp \left(\frac{3}{2}\left(1-\frac{1}{r}\right)\right)$. We denote by BB2.3 the bound Eq. 26 for $i_{0}=3$ combined with the bound Eq. 41 .

\section{B. Conjecture of a stricter bound on virial coefficients}

We now set $p=p(i)=2 i / v$ and assume that $v, n-i$ are large. From Eq. 21 16 15 it follows that for $k \geq 2$

$$
\Delta^{k}\left(\frac{1}{v} \ln (N(i) i !) \approx-\Delta^{k} \sum_{j=2}^{\infty} \frac{m_{j} p^{j}}{j-1}\right.
$$

from which it follows that

$$
\Delta^{k}\left(\frac{1}{v} \ln (N(i) i !(n-i) !) \approx \Delta^{k} \sum_{j=2}^{\infty}\left(\frac{1}{2 j}-m_{j}\right) \frac{p^{j}}{j-1}\right.
$$


All the known virial coefficients in the infinite regular lattice models mentioned in the introduction satisfy the bound

$$
m_{k} \geq \frac{1}{2 k}
$$

for $k \geq 2$. We have also checked that, in $d$ dimensional hypercubic infinite lattices the virial expansion coefficients satisfy the bound Eq. (47) through order 20 for any $d$, so that from Eq. (46) it follows that Eq. (2) is satisfied in these cases. We are therefore lead to conjecture that Eq. (47) holds on all infinite regular lattices. This indicates that there is a singularity in the virial expansion of the pressure, for $p=1$. As already indicated in [10] the entropy is analytic in the interval $0 \leq p<1$ and it is bounded above at $p=1$. However from Eq. (7) if follows that $\frac{d \lambda}{d p}$ is $\log$-singular for large $z$ (equivalently for $p \rightarrow 1$ ). As to the pressure, from Eq. (6) and the fact that $\lambda(p)$ is bounded above at $p=1$ and $p(z) \rightarrow 1$ as $z \rightarrow \infty$, it follows that the pressure is log-singular for large $z$.

Eq. (2) for $k=2$ is satisfied for any graph, due to the Heilmann-Lieb inequality Eq. (23). We are therefore led to the conjecture that in all infinite regular lattice models the virial coefficients satisfy Eq. (47), and to the conjecture that the frequency of the violations to the bounds Eq. (2) for large regular biconnected graphs, tends to zero as $v \rightarrow \infty$.

In [19], 25] it has been conjectured that in the limit of infinitely large random bipartite regular graphs, the entropy has almost surely the coefficients $a_{k}$ given in Eq. (13); the same is true for the average distribution Eq. 49 ) introduced in [19. In the appendix we show that this average distribution satisfies the bounds Eq. 22), so that this conjecture suggests that the bipartite regular graphs almost surely satisfy Eq. (22).

In the tests discussed in the next section, no violation of the bound Eq. (2) with $k=3$ are observed both for regular biconnected graphs and for not necessarily regular lattice graphs. Notice however that for $k=3$, in the case of more general non-regular graphs there are violations, e.g. in the case of the graph with perfect matching shown in Figure 1.

It seems therefore interesting to investigate the bound Eq. (2) for $k=3$ in the case of biconnected regular graphs. Using Eq. (53) for $k=3$ we get

$$
P(i) \leq P\left(i_{0}\right)\left(\frac{P\left(i_{0}+1\right)}{P\left(i_{0}\right)}\right)^{i-i_{0}}\left(\frac{P\left(i_{0}+2\right) P\left(i_{0}\right)}{P\left(i_{0}+1\right)^{2}}\right)^{\frac{\left(i-i_{0}\right)\left(i-i_{0}-1\right)}{2}}
$$

for $i \geq i_{0}$.

In the case of regular graphs, we can use Eq. (48) with $i_{0}=0$, taking into account Eq. 42 for $N(2)$. The conjectured bound BR3.0c is obtained combining this bound with Eq. (38).

As an example of a regular graph, consider the Buckminster fullerene $C_{60}$; it has $v=60$ and $E=90$; the first violation of Eq. (2) is for $k=20$. The logarithm of the Hosoya index $Z$ of this graph has the value $\ln (Z)=34.89$. The bound BR has the value 46.49, the bound BR2.0 yields 41.50, the bound BR2.1 is 41.02, while BR3.0c is 36.58.

In the case of regular bipartite graphs, we can use Eq. (48) with $i_{0}=2$, using $N(2), N(3)$ and replacing $N(4)$ by $N_{m}(4)$, as in subsection IIIA. We thus conjecture a bound (called BB3.2c) obtained combining this bound with Eq. 41.

When $r$ divides $n$, the bound BB3.2c is weaker than the Upper Matching conjecture (UMC) [19, according to which the number of $i$-matchings of a $r$-regular bipartite graph with $n=q r$ is bounded above by the number of $i$-matchings of $q K_{r, r}$, for $q$ a positive integer.

In Table II we give $\ln Z$, where $Z$ is the Hosoya index, and upper bound estimates for two graphs, the periodic $12 \times 12$ square grid and the 6 -cube; for the latter we took the matching polynomial from [22] which used [23|24].

TABLE II: $\ln Z$, where $Z$ is the Hosoya index and upper bounds estimates for two bipartite regular graphs. The first graph is the periodic grid $12 \times 12$, with $V=144$ and degree 4 ; the second one is the 6 -cube, with $V=64$ and degree 6 . The latter two upper bounds BB3.2c and UMC are conjectural. The UMC conjecture applies only to graphs in which $V / 2$ is a multiple of the degree, so that for the 6-cube no entry appears in the Table.

\begin{tabular}{|c|c|c|c|c|c|}
\hline graph & $\ln Z$ & BB & BB2.3 & BB3.2c & UMC \\
\hline periodic grid $12 \times 12$ & 95.44 & 115.24 & 113.69 & 100.11 & 96.16 \\
6-cube & 50.32 & 59.59 & 58.49 & 52.87 & \\
\hline
\end{tabular}

The $d$-cubes through $d=6$ satisfy the bounds Eq. (1) and Eq. (2). 


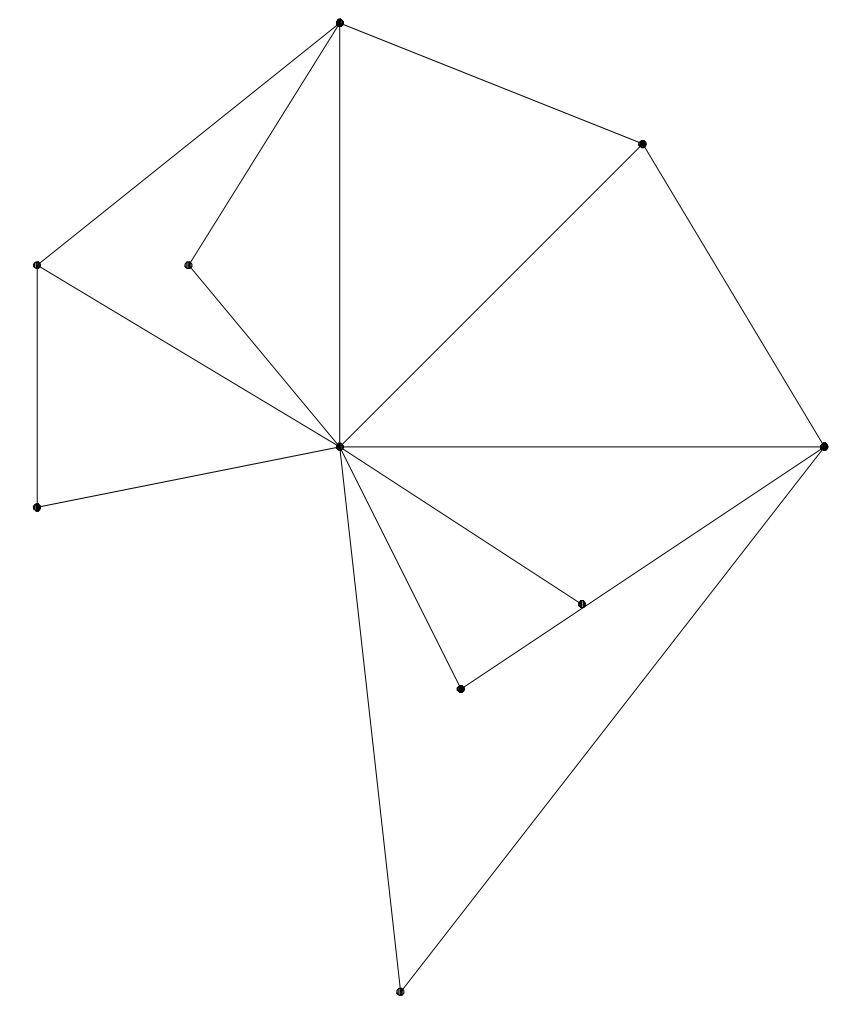

FIG. 1: A graph violating the bound Eq. 2 for $k=3$.

\section{TESTS ON THE UPPER BOUNDS}

We shall now review our graph tests. All graphs we considered are simple biconnected graphs. The tests of Eqs. (1) and Eqs.(2) are done for all allowed values of $i$ (one could check only the case $i=0$ to see if there are violations for some $k$, but the value of $k$ for which there is a violation is generally higher if one considers only the case $i=0$ ).

We made tests on lattice graphs and on regular graphs; to generate systematically the latter we have used the geng program in the Nauty package[15, via the Sage interface [16]. The matching generating polynomials are computed with the aid of the algorithm described in 33. To perform our computations, we have used an ordinary desktop personal computer based on a processor Intel $i 7860$ with a RAM of $8 G B$. 


\section{A. Tests of the bounds Eq. (1) on finite lattice graphs. i) Periodic boundary conditions}

Let us first discuss the tests on finite lattices with periodic $b c$. In the case of rectangular grids of size $L_{x} \times L_{y}$ with $L_{x} \geq L_{y}$, we considered the cases listed in Tab. III

TABLE III: Violations of the bounds Eq. (1) in the case of rectangular grids of size $L_{x} \times L_{y}$, with $L_{x} \geq L_{y}$ and periodic $b c$.

\begin{tabular}{|c|c|c|}
\hline$L_{x}$ & $L_{y}$ & Violations of the bounds Eq. 2 \\
\hline$L_{x} \leq 2000$ & $L_{y}=3$ & for $L_{x} \geq 9$ \\
$L_{x} \leq 1100$ & $L_{y}=4$ & for all $L_{x} \geq 409$ \\
$L_{x} \leq 500$ & $L_{y}=5$ & for $L_{x} \geq 186$ \\
$L_{x} \leq 200$ & $L_{y}=6$ & none \\
$L_{x} \leq 70$ & $L_{y}=7$ & none \\
$L_{x} \leq 50$ & $L_{y}=8$ & none \\
$L_{x} \leq 20$ & $L_{y}=9$ & none \\
$L_{x} \leq 15$ & $L_{y}=10$ & none \\
$L_{x}=11$ & $L_{y}=11$ & none \\
$L_{x}=12$ & $L_{y}=12$ & none \\
\hline
\end{tabular}

Therefore we have found violations only for $L_{y} \leq 5$, when $L_{x} \geq 3 L_{y}$. The minimum $k$ for which there are violations is 12 for the cases with $L_{y}=3$, it is larger than 100 in the other cases.

In the case of triangular grids of size $L_{x} \times L_{y}$ with $L_{x} \geq L_{y}$, we have considered the cases listed in Tab. IV.

TABLE IV: Violations of the bounds Eq. (1) in the case of triangular grids of size $L_{x} \times L_{y}$, with $L_{x} \geq L_{y}$ and periodic $b c$. These grids are obtained by adding a SW-NE diagonal to rectangular grids $L_{x} \times L_{y}$.

\begin{tabular}{|c|c|c|}
\hline$L_{x}$ & $L_{y}$ & Violations of the bounds Eq. 2 \\
\hline$L_{x} \leq 1000$ & $L_{y}=3$ & for all $L_{x} \geq 16$ \\
$L_{x} \leq 1000$ & $L_{y}=4$ & none \\
$L_{x} \leq 400$ & $L_{y}=5$ & for $L_{x} \geq 66$ \\
$L_{x} \leq 200$ & $L_{y}=6$ & none \\
$L_{x} \leq 50$ & $L_{y}=7$ & none \\
$L_{x} \leq 17$ & $L_{y}=8$ & none \\
\hline
\end{tabular}

In the case of hexagonal lattices of size $L_{x} \times L_{y}$ with periodic $b c$ (in the brick-wall representation) with $L_{x}, L_{y}$ even, we have considered the cases listed in Tab. V

TABLE V: Violations of the bounds Eq. (1) in the case of hexagonal grids of size $L_{x} \times L_{y}$, with $L_{x} \geq L_{y}$ and periodic $b c$.

\begin{tabular}{|c|c|c|}
\hline$L_{x}$ & $L_{y}$ & Violations of the bounds Eq. 2 \\
\hline$L_{x}=4$ & $4 \leq L_{y} \leq 100$ & all cases \\
$L_{x}=6$ & $4 \leq L_{y} \leq 100$ & for $L_{y} \geq 26$ \\
$8 \leq L_{x} \leq 14$ & $4 \leq L_{y} \leq 14$ & none \\
$6 \leq L_{x} \leq 100$ & $L_{y}=4$ & none \\
$6 \leq L_{x} \leq 100$ & $L_{y}=6$ & none \\
\hline
\end{tabular}

The minimum value of $k$ for which we found a violation is 7 . In particular there are no violations for $L_{x}$ close to $L_{y}$ and $L_{x}>4$.

\section{B. Tests of the bounds Eq. (1) on finite lattice graphs. ii) Open boundary conditions}

Let us now turn to finite lattices with open boundary conditions.

In the case of rectangular grids of size $L_{x} \times L_{y}$, with open $b c$, we have examined the cases $2 \leq L_{y} \leq L_{x} \leq 19$, finding no violations to Eq. (1). 
In the case of triangular grids of size $L_{x} \times L_{y}$, obtained by adding a SW-NE diagonal in a rectangular grids $L_{x} \times L_{y}$ with open $b c$, for $2 \leq L_{y} \leq L_{x} \leq 18$ there are violations only for $L_{y} \times 3$ with $L_{x} \geq 10$. The minimum value for which Eq. (1) is violated is $k=16$.

We have also examined three-dimensional slabs of size $L_{x} \times L_{y} \times L_{z}$ with open $b c$. The results are summarized in Tab. VI.

TABLE VI: Violations of the bounds Eq. (1) in the case of three-dimensional slabs of size $L_{x} \times L_{y} \times L_{z}$ with open $b c$.

\begin{tabular}{|c|c|c|c|}
\hline$L_{x}$ & $L_{y}$ & $L_{z}$ & Violations of the bounds Eq. 22 \\
\hline $2 \leq L_{x} \leq 1000$ & $L_{y}=3$ & $L_{z}=2$ & none \\
$3 \leq L_{x} \leq 500$ & $L_{y}=3$ & $L_{z}=3$ & none \\
$4 \leq L_{x} \leq 600$ & $L_{y}=4$ & $L_{z}=2$ & none \\
$4 \leq L_{x} \leq 200$ & $L_{y}=4$ & $L_{z}=3$ & none \\
$4 \leq L_{x} \leq 40$ & $L_{y}=4$ & $L_{z}=4$ & none \\
\hline
\end{tabular}

For all the graphs examined in this section, Eq. (1) is satisfied for $k \leq 4$. It would be interesting to know whether these bounds, Eq. (1) for $k \leq 4$, are always satisfied for regular biconnected graphs.

\section{Tests of the bounds Eq. (1) on bipartite graphs}

We have tested the validity of the upper bounds Eq. (18) (equivalently Eq. (1)) for regular bipartite biconnected graphs (RBB), by enumerating and studying exhaustively a large class of graphs. For $v \leq 18$ vertices, we observe a single violation in the case of a 3-regular graph with $v=18$. For 3-regular graphs with $18 \leq v \leq 30$, the frequency of the violations decreases with increasing $v$ ( $v$ is even, since there are no graphs with $v$ odd in this class).

It is interesting to observe that, in the cases considered in TableVII the average order of the automorphism groups of the positivity-violating graphs is a few times larger than the average order of the automorphism groups of all the $\mathrm{RBB}$ graphs with the same vertex degree. The same is true in the following tests of this section.

TABLE VII: For the RBB graphs with a given number $18 \leq v \leq 30$ of vertices of degree 3 , we have listed the number of graphs in this class, the number of violations of the upper bounds, the average order $n g$ of the automorphism groups of the graphs, the average order $n g v$ of this group for the graphs violating the bounds Eq. (1). $k$ is the minimum value for which Eq. (1) is violated.

\begin{tabular}{|c|c|c|c|c|c|}
\hline$v$ & number of graphs & violations & $n g$ & $n g v$ & $k$ \\
\hline 18 & 149 & 1 & 15.1 & 64. & 9 \\
20 & 703 & 3 & 8.7 & 91. & 10 \\
22 & 4132 & 13 & 4.5 & 40. & 9 \\
24 & 29579 & 38 & 3.3 & 32. & 7 \\
26 & 245627 & 253 & 2.3 & 22. & 7 \\
28 & 2291589 & 1392 & 1.9 & 20. & 6 \\
30 & 23466857 & 8008 & 1.7 & 16. & 7 \\
\hline
\end{tabular}

For the RBB graphs with vertices of degree 4 there is one violation for $v=20$ among 62611 graphs and $k=10$ is the minimum value for which Eq. (1) is violated. The order of the automorphism group of the violating graph is 256, while the average order is 3.1. For $v=22$, there are 5 violations among 2806490 graphs and the minimum value for which Eq. (1) is violated is $k=11$; the average of the orders of the automorphism groups of the violating graphs is 3721.6 , while the average order of all graphs is 1.5 .

For the RBB graphs with vertices of degree 5 , there is one violation for $v=20$ out of 304495 graphs, with $k=9$; the order of the automorphism group of this graph is 1327104, while the average order is 7.1.

For the RBB graphs with vertices of degree larger than 4, we had to restrict to graphs with $v \leq 20$ vertices and observed no violations for degree larger than 5. 


\section{Tests of the bounds Eq. (1) on non-bipartite graphs}

In the case of the biconnected 3-regular non-bipartite graphs with $v \leq 22$, the first violation occurs for $v=12$. For $v \geq 12$ the frequency of the violations decreases with $v$, as shown in Table VIII.

TABLE VIII: For the biconnected 3-regular non-bipartite graphs with a given number $v$ of vertices, we have listed the number of graphs in this class, the number of violations of the upper bounds, the average order $n g$ of the automorphism groups of the graphs, the average order $n g v$ of this group for the graphs violating the bounds Eq. (1). The cases with $v$ odd are not listed, since there are no such graphs. $k$ is the minimum value for which Eq. (1) is violated.

\begin{tabular}{|c|c|c|c|c|c|}
\hline$v$ & number of graphs & violations & $n g$ & $n g v$ & $k$ \\
\hline 12 & 76 & 1 & 7.4 & 16. & 6 \\
14 & 467 & 6 & 4.4 & 8.7 & 6 \\
16 & 3836 & 44 & 3.1 & 8.3 & 5 \\
18 & 39717 & 257 & 2.2 & 7.5 & 5 \\
20 & 497115 & 2856 & 1.7 & 5.6 & 5 \\
22 & 7183495 & 29597 & 1.5 & 4. & 5 \\
\hline
\end{tabular}

In the case of the biconnected 4-regular non-bipartite graphs with $v \leq 17$, the first violations occur for $v=12$, as shown in Table IX

TABLE IX: For the biconnected 4-regular non-bipartite graphs with a given number $v$ of vertices, we have listed the number of graphs in this class, the number of violations of the upper bounds, the average order $n g$ of the automorphism groups of the graphs, the average order $n g v$ of this group for the graphs violating the bounds Eq. (1). $k$ is the minimum value for which Eq. (1) is violated.

\begin{tabular}{|c|c|c|c|c|c|}
\hline$v$ & number of graphs & violations & $n g$ & $n g v$ & $k$ \\
\hline 12 & 1538 & 2 & 3.4 & 40 & 6 \\
13 & 10768 & 0 & & & \\
14 & 88112 & 12 & 1.6 & 17 & 6 \\
15 & 805281 & 30 & 1.3 & 14. & 7 \\
16 & 8036122 & 454 & 1.2 & 14. & 6 \\
17 & 86214189 & 295 & 1.2 & 10. & 6 \\
\hline
\end{tabular}

Unlike in the bipartite case and in the case of 3-regular non-bipartite graphs examined above, there exist graphs with odd $v$.

The frequency of the violations occurring among the graphs with even $v$ decreases regularly as $v$ increases. The same is true for the graphs with odd $v$.

\section{E. Tests of the bounds Eq. (2) on finite lattice graphs. i) Periodic boundary conditions}

Let us first discuss the tests on finite lattices with periodic $b c$.

In the case of rectangular grids of size $L_{x} \times L_{y}$ with $L_{x} \geq L_{y}$, we have considered the cases listed in Tab. X] 
TABLE X: Violations of the bounds Eq. 2) in the case of rectangular grids of size $L_{x} \times L_{y}$, with $L_{x} \geq L_{y}$ and periodic $b c$.

\begin{tabular}{|c|c|c|}
\hline$L_{x}$ & $L_{y}$ & Violations of the bounds Eq. 2 \\
\hline$L_{x} \leq 2500$ & $L_{y}=3$ & for all $L_{x} \geq 5$ \\
$L_{x} \leq 1000$ & $L_{y}=4$ & for most $L_{x} \geq 8$ \\
$L_{x} \leq 700$ & $L_{y}=5$ & for most $L_{x} \geq 9$ \\
$L_{x} \leq 200$ & $L_{y}=6$ & for most $L_{x} \geq 12$ \\
$L_{x} \leq 70$ & $L_{y}=7$ & none \\
$L_{x} \leq 50$ & $L_{y}=8$ & for most $L_{x} \geq 14$ \\
$L_{x} \leq 20$ & $L_{y}=9$ & none \\
$L_{x} \leq 15$ & $L_{y}=10$ & none \\
$L_{x}=11$ & $L_{y}=11$ & none \\
$L_{x}=12$ & $L_{y}=12$ & none \\
\hline
\end{tabular}

The minimum value of $k$ for which violations are observed, is 4 .

In the case of the triangular grids of size $L_{x} \times L_{y}$ with $L_{x} \geq L_{y}$ and periodic $b c$, we considered the cases listed in $\mathrm{Tab} \mathrm{XI}$

TABLE XI: Violations of the bounds Eq. 22 in the case of triangular grids of size $L_{x} \times L_{y}$, with $L_{x} \geq L_{y}$ and periodic $b c$.

\begin{tabular}{|c|c|c|}
\hline$L_{x}$ & $L_{y}$ & Violations of the bounds Eq. \\
\hline$L_{x} \leq 2500$ & $L_{y}=3$ & for most $L_{x} \geq 11$ \\
$L_{x} \leq 1000$ & $L_{y}=4$ & for all $L_{x} \geq 88$ \\
$L_{x} \leq 500$ & $L_{y}=5$ & for most $L_{x} \geq 29$ \\
$L_{x} \leq 150$ & $L_{y}=6$ & none \\
$L_{x} \leq 50$ & $L_{y}=7$ & none \\
$L_{x} \leq 15$ & $L_{y}=8$ & none \\
\hline
\end{tabular}

The minimum value of $k$ for which there are violations is 4 .

In the case of the hexagonal grids (in the brick-wall representation) of size $L_{x} \times L_{y}$, with $L_{x} \geq L_{y}$ and periodic $b c$ we considered the cases listed in TabXII.

TABLE XII: Hexagonal grids (in the brick-wall representation) of size $L_{x} \times L_{y}$, with $L_{x} \geq L_{y}$ and periodic $b c$ tested .

\begin{tabular}{|c|c|c|}
\hline$L_{x}$ & $L_{y}$ & violations of the bound Eq. 2 \\
\hline$L_{x}=4$ & $4 \leq L_{y} \leq 100$ & $L_{y}=6$ and $L_{y}>=10$ \\
$L_{x}=6$ & $4 \leq L_{y} \leq 100$ & $L_{y} \geq 6$ \\
$8 \leq L_{x} \leq 14$ & $4 \leq L_{y} \leq 14$ & none for $L_{x}-8 \leq L_{y}<L_{x}$ \\
$8 \leq L_{x} \leq 100$ & $L_{y}=4$ & $L_{y} \geq 14$ \\
$8 \leq L_{x} \leq 30$ & $L_{y}=6$ & $L_{x} \geq 18$ \\
\hline
\end{tabular}

Summarizing this table, we found no violations in the band $\max \left(4, L_{x}-8\right) \leq L_{y}<L_{x}$; almost all the other cases violate the bounds. The violations of the bounds Eq. (2) occur for $k \geq 4$.

\section{F. Tests of the bounds Eq. (2) on finite lattice graphs. ii) Open boundary conditions}

Let us now turn to finite lattices with open $b c$.

In the case of the rectangular grids of size $L_{x} \times L_{y}$ with $2 \leq L_{y} \leq L_{x} \leq 19$ and open $b c$, there are many violations, but they do not occur for $L_{x}=L_{y}$ with $L_{x} \neq 5$, or for $L_{y} \geq 9$. The minimum value of $k$ for which Eq. (2) is violated is $k=4$.

In the case of the triangular grids $L_{x} \times L_{y}$ with $2 \leq L_{y} \leq L_{x} \leq 18$ and open $b c$, there are no violations for $L_{x}=L_{y}$ with $L_{x} \neq 5$, or for $L_{y} \geq 8$. The minimum $k$ for which there are violations to Eq. (2) is $k=6$.

In the case of the 3 -d grids of size $L_{x} \times L_{y} \times L_{z}$ with open $b c$, we considered the cases listed in Tab. XIII. 
TABLE XIII: Violations of the bounds Eq. 22 in the case of three-dimensional slabs of size $L_{x} \times L_{y} \times L_{z}$ with open $b c$.

\begin{tabular}{|c|c|c|c|}
\hline$L_{x}$ & $L_{y}$ & $L_{z}$ & Violations of the bounds Eq. 2 \\
\hline$L_{x} \leq 1000$ & $L_{y}=3$ & $L_{z}=2$ & for $L_{x} \geq 8$ \\
$L_{x} \leq 500$ & $L_{y}=3$ & $L_{z}=3$ & for $L_{x}=5,9,11,13,15,17,21$ \\
$L_{x} \leq 600$ & $L_{y}=4$ & $L_{z}=2$ & for $L_{x} \geq 11$ \\
$L_{x} \leq 200$ & $L_{y}=4$ & $L_{z}=3$ & for $L_{x} \geq 16$ \\
$L_{x} \leq 40$ & $L_{y}=4$ & $L_{z}=4$ & for $L_{x} \geq 20$ \\
\hline
\end{tabular}

In particular, no violations are observed for $L_{x}=L_{y}=L_{z}$.

Summarizing, in the tests of Eq. 22 on lattice graphs, violations are observed more often than for Eq. (1); for bidimensional lattices with $L_{x} \approx L_{y}>5$ there are no violations, except in the case of the hexagonal lattice, in which no violations are present on a strip with $L_{x}>L_{y}$. This restriction might be due to the fact that the hexagonal lattice is sensitive to the boundary conditions 34]. Therefore these tests give some indication, although not as sharply as in the case of Eq. (1), that in the limit of infinite lattices the bounds Eq. (2) and the corresponding bounds Eq. (47) are satisfied, and virial positivity holds.

\section{G. Tests of the bounds Eq. (2) on bipartite graphs}

Let us now discuss the systematic tests on regular graphs.

In the case of the RBB graphs with vertices of degree 3 and $v \leq 30$, the first violation occurs for $v=14$; for larger values of $v$ the frequency of violations decreases irregularly (e.g. it increases at $v=20$ ), as shown in Table XIV]

TABLE XIV: For the RBB graphs with a given number $14 \leq v \leq 30$ of vertices of degree 3 , we have listed the number of graphs in this class, the number of violations of the upper bounds Eq. (2), the average order $n g$ of the automorphism groups of the graphs, the average order $n g v$ of this group for the graphs violating the bounds Eq. (2); $k$ is the minimum value for which these bounds are violated.

\begin{tabular}{|c|c|c|c|c|c|}
\hline$v$ & number of graphs & violations & $n g$ & $n g v$ & $k$ \\
\hline 14 & 13 & 1 & 44. & 28. & 7 \\
16 & 38 & 2 & 19. & 48. & 6 \\
18 & 149 & 5 & 15. & 84. & 5 \\
20 & 703 & 33 & 8.7 & 29. & 5 \\
22 & 4132 & 106 & 4.5 & 20. & 4 \\
24 & 29579 & 619 & 3.3 & 16. & 4 \\
26 & 245627 & 3415 & 2.3 & 10. & 4 \\
28 & 2291589 & 22913 & 1.9 & 7.9 & 4 \\
30 & 23466857 & 163789 & 1.7 & 6.2 & 4 \\
\hline
\end{tabular}

In the case of the RBB graphs with vertices of degree 4 and $v \leq 22$, the first violation occurs for $v=16$. For larger values of $v$ the frequency of the violations decreases, as shown in the Table $\mathrm{XV}$.

In the case of RBB graphs with vertices of degree 5 and $v \leq 20$, the first 3 violations occur for $v=20$ out of 304495 graphs, with average order 449280 of the automorphism groups of the violating graphs, to be compared with an average order of 7.1 for all the graphs; the minimum $k$ for which there are violations is $k=6$.

We have checked that for RBB graph with $v \leq 20$, there are no violations for $v>5$.

\section{H. Tests of the bounds Eq. (2) on non-bipartite graphs}

In the case of biconnected 3-regular non-bipartite graphs with $v \leq 20$, the first violation occurs for $v=10$. For larger values of $v$ the frequency of violations decreases irregularly as shown in Table XVI.

In the case of biconnected 4-regular non-bipartite graphs with $v \leq 17$, a first violation is met for $v=11$. For larger $v$, the frequency of the violations decreases regularly for $v$ even, while it decreases irregularly for $v$ odd (it increases for $v=15$ ), see Table XVII.

In addition to the systematic examination of 3-regular biconnected graphs up to $v=22$, we have tested the $k=3$ bound for $v=30$. Using the NetworkX 35] random regular graph generator, we have thus examined over 100 millions 
TABLE XV: For the RBB graphs with $16 \leq v \leq 22$ vertices of degree 4 , we have listed the number of graphs in this class, the number of violations of the upper bounds Eq. (2), the average order $n g$ of the automorphism groups of the graphs, the average order $n g v$ of this group for the graphs violating the bounds Eq. [2]; $k$ is the minimum value for which these bounds are violated.

\begin{tabular}{|c|c|c|c|c|c|}
\hline$v$ & number of graphs & violations & $n g$ & $n g v$ & $k$ \\
\hline 16 & 129 & 1 & 112. & 5184. & 8 \\
18 & 1980 & 1 & 8.7 & 576. & 7 \\
20 & 62611 & 18 & 3.1 & 1901. & 6 \\
22 & 2806490 & 115 & 1.5 & 487. & 6 \\
\hline
\end{tabular}

TABLE XVI: For the biconnected non-bipartite graphs with a given number $10 \leq v \leq 22$ of vertices of degree 3 , we have listed the number of graphs in this class, the number of violations of the upper bounds Eq. (2), the average order $n g$ of the automorphism groups of the graphs, the average order $n g v$ of this group for the graphs violating the bounds Eq. (2); $k$ is the minimum value for which these bounds are violated.

\begin{tabular}{|c|c|c|c|c|c|}
\hline$v$ & number of graphs & violations & $n g$ & $n g v$ & $k$ \\
\hline 10 & 16 & 1 & 15. & 16. & 5 \\
12 & 76 & 2 & 7.4 & 12. & 5 \\
14 & 467 & 11 & 4.4 & 11. & 4 \\
16 & 3836 & 102 & 3.1 & 9. & 4 \\
18 & 39717 & 741 & 2.2 & 6.2 & 4 \\
20 & 497115 & 7324 & 1.7 & 4.8 & 4 \\
22 & 7183495 & 78426 & 1.5 & 3.5 & 4 \\
\hline
\end{tabular}

3-regular graphs with $v=30$, and have checked that Eq. (2) is satisfied for $k=3$. From Table XVI, by a simple extrapolation we estimate that for $v=30$ there are roughly $8 \times 10^{11}$ graphs, so the fraction of non-inequivalent produced random graphs is expected to be less than $10^{-4}$.

Analogously, we have examined 100 millions random 4-regular biconnected graphs with 21 vertices. We have checked that Eq. (2) is satisfied for $k=3$. From Table XVII we estimate that for $v=21$ there are roughly $2 \times 10^{12}$ graphs, so the fraction of equivalent random graphs produced by the generator, is expected to be less than $10^{-4}$.

In all the tests performed on regular graphs (over more than 300 million graphs) no violations of Eq. (2) for $k=3$ are observed.

As a further comment, we observe that there are some similarities between the graph positivity property 14 and Eq. (1): in both cases the violating graphs have an average order of the automorphism groups which is several times larger than the average over all the graphs with the same number of vertices $v$. This property is observed also in the case of Eq. [2], but to a lesser extent.

\section{CONCLUSIONS}

We have observed that the coefficients of the virial expansions of the MD model computed up to now satisfy Eq. 47) on all infinite regular lattices. In particular the first 20 coefficients of the virial expansion satisfy Eq. (47) for the hypercubic infinite lattices of any dimension $d$. This led us to conjecture that the virial coefficients are all positive for any infinite regular lattice model, and to the stricter conjecture that they all satisfy Eq. (47).

Using a simple relation between the virial coefficients and the coefficients of the series for the dimer entropy, the conjecture on the positivity of the virial coefficients led us to test the validity of the bounds in Eq. (1) for the finite lattice graphs and also for the finite graphs which somehow generalize them, namely the biconnected regular graphs.

For $k=2$ these bounds follow from the inequality Eq. (23) proved by Heilmann and Lieb.

We have shown that this inequality leads to rigorous upper bounds for the number of matchings $N(i)$ improving those known up to now for regular graphs 32 in the region of low dimer density, and for general graphs. In the latter case, we derive an upper bound for the matching matching polynomial improving the one in [18].

The tests on lattice graphs support the validity of the virial positivity conjecture.

Our tests on a large class of regular graphs and lattice graphs also support the conjecture that the frequency of violations of Eq. (1) tends to zero as $v \rightarrow \infty$.

We have proposed the more general bounds Eq. (2) corresponding to the stricter conjecture that Eq. 47) is valid for infinite regular lattices . 
TABLE XVII: For the biconnected non-bipartite graphs with $10 \leq v \leq 17$ vertices of degree 4 , we have listed the number of graphs in this class, the number of violations of the upper bounds Eq. $\overline{2}$, the average order $n g$ of the automorphism groups of the graphs, the average order $n g v$ of this group for the graphs violating the bounds Eq. (2); $k$ is the minimum value for which these bounds are violated.

\begin{tabular}{|c|c|c|c|c|c|}
\hline$v$ & number of graphs & violations & $n g$ & $n g v$ & $k$ \\
\hline 11 & 264 & 1 & 5.2 & 12. & 5 \\
12 & 1538 & 3 & 3.4 & 32. & 5 \\
13 & 10768 & 37 & 2.1 & 16. & 5 \\
14 & 88112 & 34 & 1.6 & 31. & 5 \\
15 & 805281 & 3086 & 1.3 & 5.0 & 4 \\
16 & 8036122 & 1121 & 1.2 & 14. & 5 \\
17 & 86214189 & 197431 & 1.2 & 2.5 & 4 \\
\hline
\end{tabular}

Our tests of the bounds Eq. (2) on the regular graphs give results similar to those obtained for the bounds Eq. (1), although with more violations.

The tests of Eq. (2) on lattice graphs show many more violations than for the bounds Eq. (1); there are however bands with $L_{x} \approx L_{y}$ in the $2 \mathrm{~d}$ case and with $L_{x} \approx L_{y} \approx L_{z}$ for the cubic slabs, for which there are no violations. Extrapolating the behavior in these regions to the limit of large lattices we get some indication that there are no violations of bounds Eq. (2) and the inequality Eq. (47) in the infinite lattice limit, but this indication is not as strong as in the case of the bounds Eq. (1) and virial positivity.

In all tests (carried out over more than 300 million regular graphs), we found no violations of the bounds Eq. (2) for $k=3$. It would be interesting to know whether the bounds Eq. (2) for $k=3$ are satisfied by all regular biconnected graphs.

We have discussed upper bounds that could be proved if Eq. (2) held for $k=3$ : they are tighter than those we proved based on the truth of the $k=2$ case.

\section{APPENDIX A: VIRIAL COEFFICIENTS IN $d=2,3$}

The virial coefficients $m_{k}$ in $d=2,3,4$ can be obtained from the entropy coefficients $a_{k}$ computed in [10] through order 24; they satisfy the bound Eq.(47). In Table ?? we give the virial coefficients in $d=2,3$. 
TABLE XVIII: Virial coefficients $m_{k}$ in $d=2,3$ up to order 24 .

\begin{tabular}{|c|c|c|}
\hline$k$ & $d=2$ & $d=3$ \\
\hline 2 & $7 / 16$ & $11 / 24$ \\
3 & $31 / 96$ & $71 / 216$ \\
4 & $121 / 512$ & $419 / 1728$ \\
5 & $471 / 2560$ & $31 / 160$ \\
6 & $1867 / 12288$ & $15031 / 93312$ \\
7 & $7435 / 57344$ & $89951 / 653184$ \\
8 & $29477 / 262144$ & $539963 / 4478976$ \\
9 & $116383 / 1179648$ & $3244127 / 30233088$ \\
10 & $459517 / 5242880$ & $19482611 / 201553920$ \\
11 & $1821051 / 23068672$ & $116960471 / 1330255872$ \\
12 & $7255915 / 100663296$ & $702028151 / 8707129344$ \\
13 & $29063919 / 436207616$ & $1404544085 / 18865446912$ \\
14 & $16697149 / 268435456$ & $151861682911 / 2350924922880$ \\
15 & $157001097 / 2684354560$ & $197399821995527 / 3859278353399808$ \\
16 & $1898046421 / 34359738368$ & $395010395194633 / 8124796533473280$ \\
17 & $7634823999 / 146028888064$ & $2371215487550117 / 51186218160881664$ \\
18 & $30619146937 / 618475290624$ & $3882036150042265 / 87747802561511424$ \\
19 & $122399296903 / 2611340115968$ & $256340050245714583 / 6054598376744288256$ \\
20 & $488028559661 / 10995116277760$ & $1538798075750480935 / 37907050706572935168$ \\
21 & $1943383170991 / 46179488366592$ & \\
22 & $7740357251909 / 193514046488576$ & \\
23 & $30871937807467 / 809240558043136$ & \\
24 & $123369796036139 / 3377699720527872$ & \\
\hline
\end{tabular}




\section{APPENDIX B: PROOF OF EQS.(1, 2) FOR SOME CLASSES OF GRAPHS}

Let

$$
A(a, v, k)=\Delta^{k} \ln \left(N(i) i !((n-i) !)^{a}\right)
$$

with $n=\left[\frac{v}{2}\right]$ and $k \geq 2$. The bound Eq. (1) is $A(0, v, k) \leq 0$, the bound Eq. (2) is $A(1, v, k) \leq 0$.

In the case of polygons we have

$$
N(i)=\frac{v}{v-i}\left(\begin{array}{c}
v-i \\
i
\end{array}\right)
$$

and thus

$$
A(a, v, k)=\Delta^{k-1}\left(\ln \left(1-\frac{2 i}{v}\right)+\ln \left(1-\frac{2 i+1}{v}\right)-\ln \left(1-\frac{i+1}{v}\right)-a \ln \left(1-\frac{i}{n}\right)\right)
$$

For $a=0$ and all $v$ or $a=1$ and $v$ even,

$$
A(a, v, k)=\Delta^{k-1} \sum_{h=1} \frac{1}{j v^{j}}\left((i+1)^{j}-(2 i+1)^{j}-(1-a) 2^{j}\right)<0
$$

In the case of the complete graphs $K_{v}$, we have

$$
N(i)=\frac{v !}{(v-2 i) ! i ! 2^{i}}
$$

and thus

$$
A=\Delta^{k-1}\left(\ln \left(1-\frac{2 i}{v}\right)+\ln \left(1-\frac{2 i+1}{v}\right)-a \ln \left(1-\frac{i}{n}\right)\right)
$$

For $a=0$ and all $v$ or $a=1$ and even $v$ one gets

$$
A=\Delta^{k-1} \sum_{j=1} \frac{1}{j v^{j}}\left(-(1-a)(2 i)^{j}-(2 i+1)^{j}\right)<0
$$

In the case of the complete bipartite graphs $K_{n, n}$, we have

$$
N(i)=\left(\begin{array}{c}
n \\
i
\end{array}\right)^{2} i !
$$

and thus

$$
A(a, v, k)=(2-a) \Delta^{k-1} \ln \left(1-\frac{i}{n}\right)<0
$$

Friedland, Krop and Markström[19] have computed an average distribution for $N(i)$ in the case of random regular bipartite graphs

$$
N(i)=\frac{\left(\begin{array}{l}
n \\
i
\end{array}\right)^{2} r^{2 i} i !(r n-i) !}{(r n) !}
$$

and thus

$$
A=\Delta^{k-1}\left((2-a) \ln \left(1-\frac{i}{n}\right)-\ln \left(1-\frac{i}{r n}\right)\right)=\Delta^{k-1} \sum_{J=1} \frac{1}{j}\left(-(2-a)\left(\frac{i}{n}\right)^{j}+\left(\frac{i}{r n}\right)^{j}\right)<0
$$

The easily derived bipartite mean-field approximation

$$
N(i)=\left(\begin{array}{c}
n \\
i
\end{array}\right)^{2} i !\left(\frac{r}{n}\right)^{i}
$$

satisfies the bounds; the proof is similar to that for $K_{n, n}$. 


\section{APPENDIX C}

Consider the bounds

$$
\Delta^{k} g(i) \leq 0
$$

valid for some $k \geq 1$ and for $i \geq i_{0}$. Summing from $i_{0}$ to $i-1$ one gets

$$
\Delta^{k-1} g(i) \leq \Delta^{k-1} g\left(i_{0}\right)
$$

Summing again and using

$$
\sum_{k=1}^{n}\left(\begin{array}{c}
k \\
m
\end{array}\right)=\left(\begin{array}{c}
n+1 \\
m+1
\end{array}\right)
$$

we can prove by induction that, for $h \leq k$

$$
\Delta^{k-h} g(i) \leq \sum_{t=0}^{h-1}\left(\begin{array}{c}
i-i_{0} \\
t
\end{array}\right) \Delta^{k-h+t} g\left(i_{0}\right)
$$

In the case $h=k$ Eq. 52 gives

$$
g(i) \leq \sum_{t=0}^{k-1}\left(\begin{array}{c}
i-i_{0} \\
t
\end{array}\right) \Delta^{t} g\left(i_{0}\right)
$$

for $i \geq i_{0}$.

If a graph satisfies the bound Eq. (2) for a given $k$, then Eq. (53) with $g(i)=\ln P(i)$, where $P$ is defined in Eq. (25), gives an upper bound for $N(i)$. 
* Electronic address: paolo.butera@mib.infn.it

$\dagger$ Electronic address: pfed@umich.edu

¥ Electronic address: mario.pernici@mi.infn.it

1 S. McKenzie, "Extended high-temperature low-field expansions for the Ising model", Can. J. Phys. 57, 1239 (1979).

2 P.Federbush, "For the Monomer-Dimer Problem on Triangular and Hexagonal Lattices, the New $p$-Expansion", arXiv:1110.0684 DOI:2011arXiv1110.0684F

3 D.S. Gaunt, "Exact Series-Expansion Study of the Monomer-Dimer Problem", Phys. Rev. 179, 174 (1969). DOI:10.1103/PhysRev.179.174

4 D. A. Kurtze and M.E. Fisher, "Yang-Lee edge singularity at high temperature", Phys. Rev. B 20, 2785 (1979). DOI:10.1103/PhysRevB.20.2785

5 M.E. Fisher and T.N.V. Temperley, "Association Problem in Statistical Mechanics. Critique of the Treatment of H. S. Green and R. Leipnik", Revs. Mod. Phys. 32, 1029 (1960).

DOI:10.1103/RevModPhys.32.1029

${ }^{6}$ S. Friedland and U. N. Peled, "Theory of computation of multidimensional entropy with an application to the monomer-dimer problem", Adv. Appl. Math. 34, 486 (2005). DOI:10.1016/j.aam.2004.08.005

7 J.F. Nagle, "New series-expansion method for the dimer problem ", Phys. Rev. 152, 190 (1966). DOI:10.1103/PhysRev.152.190

8 J.F. Stilck and M.J. de Oliveira, "Entropy of flexible chains placed on Bethe and Husimi lattices", Phys. Rev. A 42, 5955 (1990). DOI:10.1103/PhysRevA.42.5955

9 P. Butera and M. Pernici, "Yang-Lee edge singularities from extended activity expansions of the dimer density for bipartite lattices of dimensionality $2 \leq d \leq 7$ ", Phys. Rev. E 86, 011104 (2012). DOI:10.1103/PhysRevE.86.011104

10 P. Butera, P. Federbush and M. Pernici, "Higher-order expansions for the entropy of a dimer or a monomer-dimer system on d-dimensional lattices", Phys. Rev. E 87, 062113 (2013). DOI:10.1103/PhysRevE.87.062113

11 O.J.Heilmann and E.H. Lieb, "Monomers and Dimers", Phys. Rev. Lett. 24 (1970) 1412. DOI:10.1103/PhysRevLett.24.1412

12 O.J.Heilmann and E.H. Lieb, "Theory of Monomer-Dimer systems", Commun. math. Phys. 25, 190 (1972). DOI : $10.1007 / 978-3-662-10018-9_{7}$

13 S. Friedland and U. N. Peled, "The pressure, densities and first order phase transitions associated with multidimensional SOFT", in Notions of Positivity and the Geometry of Polynomials, Trends in Mathematics, 179-220, (2011), Springer, Basel AG, arXiv:0906.5176

14 P. Butera, P. Federbush and M. Pernici, "A positivity property of the dimer entropy of graphs ", Physica A 421 208 (2015), arXiv:1409.4549 DOI:10.1016/j.physa.2014.11.033

15 B.D. McKay, Congr. Numer. 30, 4587 (1981); 10th Manitoba Conference on Numerical Mathematics and Computing (Winnipeg, 1980) http://cs.anu.edu.au/bdm/nauty/PGI. DOI:10.1006/jagm.1997.0898

16 William A. Stein et al., Sage Mathematics Software (Version 5.7), to be freely downloaded at the http://www.sagemath.org.

17 S. Friedland, "Results and open problems in matchings in regular graphs", Electronic J. of Linear Algebra 24, 18 (2012).

18 T. Carroll, D. Galvin and P. Tetali "Matchings and independent sets of a fixed size in regular graphs", Journal of Combinatorial Theory A 1161219 (2009).

DOI:10.1016/j.jcta.2008.12.008

19 S. Friedland, E. Krop and K. Markström, "On the Number of Matchings in Regular Graphs", Electron. J. Combin. 15.1, R110 (2008).

20 Peter Csikvari, "Lower matching conjecture, and a new proof of Schrijvers and Gurvitss theorems", arXiv:1406.0766

${ }^{21}$ L. Gurvits and A. Samorodnitsky, "Bounds on the permanent and some applications", arXiv:1408.0976.

22 "Triangle of nonzero (even) coefficients of the matching polynomials for the hypercube graphs $Q_{n}$ ", https://oeis.org/A192437

23 P. H. Lundow, "Enumeration of matchings in polygraphs", revised and updatev version of "Computation of the matching polynomial and the number of 1-factors in polygraphs, Department of Mathematics, Umea University, Research reports, No.12, 1996.

24 P. H. Lundow, "GrafPack", http://www.theophys.kth.se/ phl/Mathematica/

25 S. Friedland, E. Krop, P.H. Lundow and K. Markström, "On the Validations of the Asymptotic Matching Conjectures", J. Stat. Phys. 133, 513 (2008).

DOI:10.1007/s10955-008-9550-y

${ }^{26}$ P. Federbush and S. Friedland, "An Asymptotic Expansion and Recursive Inequalities for the Monomer-Dimer Problem", J. Stat. Phys. 143, 306 (2011).

DOI:10.1007/s10955-011-0170-6 
27 T. Biedl, E.D. Demaine, C.A. Duncan, R. Fleischer and S. G. Kobourov, "Tight bounds on maximal and maximum matchings", Discrete Math. 285, 7 (2004).

DOI:10.1016/j.disc.2004.05.003

28 P.E. Frenkel and P. Horvath, "Minkowski's inequality and sums of squares", arXiv:1206.5783 Central European Journal of Mathematics 12, 510 (2014). DOI:10.2478/s11533-013-0346-1

29 H. Hosoya, Bull. Chem. Soc. Japan "Topological index. A newly proposed quantity characterizing the topological nature of structural isomers of saturated hydrocarbons" 44, 2332 (1971).

30 I. Gutman "On the Hosoya index of very large molecules", MATCH Commun. Math. Comput. Chem. 23, 95 (1988).

31 L. Ilinca and J. Kahn, "Asymptotics of the Upper Matching Conjecture", Journal of Combinatorial Theory A 120976 (2013). DOI:10.1016/j.jcta.2013.01.013

32 S. Friedland, "Counting matchings in graphs with applications to the monomer-dimer models", KTH, April 16th (2008).

33 P. Butera and M. Pernici, "Sums of permanental minors using Grassmann algebra", arXiv:1406.5337, to appear on Int. J. Graph Theory App., (2015).

34 V. Elser, J. Phys. A: Math. Gen. "Solution of the dimer problem on a hexagonal lattice with boundary" 17(1984) 1509-1513. DOI:10.1088/0305-4470/17/7/018

35 Aric A. Hagberg, Daniel A. Schult and Pieter J. Swart, "Exploring network structure, dynamics, and function using NetworkX', in Proceedings of the 7th Python in Science Conference (SciPy2008), Edited by Gel Varoquaux, Travis Vaught, and Jarrod Millman, (Pasadena, CA USA), pp. 11-16, Aug. 2008. 